\title{
THE EQUATION $x^{\prime} \equiv x d-d x=b$
}

\section{N. JACOBSON}

Let $\mathfrak{A}$ be an associative algebra with a possibly infinite basis over a field $\Phi$. Then if $d$ is a fixed element in $\mathfrak{A}$, it is well known that the mapping $x \rightarrow x^{\prime} \equiv[x, d]=x d-d x$ is a derivation ${ }^{1}$ in $\mathfrak{A}$; that is,

$$
(x+y)^{\prime}=x^{\prime}+y^{\prime}, \quad(x \alpha)^{\prime}=x^{\prime} \alpha, \quad(x y)^{\prime}=x^{\prime} y+x y^{\prime}
$$

for all $x, y$ in $\mathfrak{A}$ and all $\alpha$ in $\Phi$. The constants relative to such a derivation are the elements of $\mathfrak{A}$ that commute with $d$. We shall call an element $b$ a $d$-integral if $b=a^{\prime}$ for some element $a$ in $\mathfrak{A}$, that is, if the equation $x^{\prime}=x d-d x=b$ has a solution in $\mathfrak{A}$. Clearly if $a$ is a solution of this equation then the totality of solutions is the set $\{a+c\}$ where $c$ ranges over the set of $d$-constants. In a recent paper appearing in this Bulletin, R. E. Johnson obtained a necessary and sufficient condition that an element $b$ be a $d$-integral under the assumption that $\mathfrak{A}$ is a separable algebraic division ring. ${ }^{2}$ In this note we allow $\mathfrak{A}$ to be an arbitrary algebra but we make the assumption that $d$ is an algebraic element in the sense that it satisfies a polynomial equation with coefficients in $\Phi$. We obtain a necessary condition, which is equivalent to Johnson's condition when $\mathfrak{A}$ is a division ring, that $b$ be a $d$-integral. If the minimum polynomial $\mu(\lambda)$ of $d$ is relatively prime to its derivative $\mu^{\prime}(\lambda)$, then it is easy to see that the condition is also sufficient and one may give an explicit formula for a solution of the equation $x^{\prime}=b$. If we assume that $\mathfrak{A}$ is a simple algebra satisfying the descending chain condition for left ideals then we can show that our condition is also sufficient when $\mu(\lambda)$ is a product of distinct irreducible factors in $\Phi[\lambda]$ and in certain other cases. Here, however, we do not display a solution but merely prove its existence. Our results include, of course, Johnson's result for algebraic division rings, since the minimum polynomial of an element in such a ring is irreducible. No assumption about separability is required.

In order to obtain a condition for the solvability of the equation $x^{\prime}=b$ we consider the matrices

$$
u=\left(\begin{array}{ll}
d & 0 \\
0 & d
\end{array}\right), \quad v=\left(\begin{array}{ll}
d & b \\
0 & d
\end{array}\right)
$$

Received by the editors May 19, 1944.

1. Cf. the author's paper Abstract derivation and Lie algebras, Trans. Amer. Math. Soc. vol. 42 (1937) pp. 206-224.

2 On the equation $\chi \alpha=\gamma \chi+\beta$ over an algebraic division ring, Bull. Amer. Math. Soc. vol. 50 (1944) pp. 202-208. 
in the matrix algebra $\mathfrak{A}_{2}$ of two-rowed matrices with elements in $\mathfrak{A}$. If $\boldsymbol{x}$ is any element in $\mathfrak{A}$

$$
\left(\begin{array}{rr}
1 & -x \\
0 & 1
\end{array}\right)=\left(\begin{array}{ll}
1 & x \\
0 & 1
\end{array}\right)^{-1}
$$

and the equation $x^{\prime}=x d-d x=b$ is equivalent to the matrix equation

$$
\left(\begin{array}{ll}
1 & x \\
0 & 1
\end{array}\right)\left(\begin{array}{ll}
d & 0 \\
0 & d
\end{array}\right)\left(\begin{array}{ll}
1 & x \\
0 & 1
\end{array}\right)^{-1}=\left(\begin{array}{ll}
d & b \\
0 & d
\end{array}\right)
$$

Thus if $b$ is a $d$-integral the matrices (1) are similar in $\mathfrak{A}_{2}$. We suppose now that $d$ is an algebraic element and let

$$
\phi(\lambda)=\lambda^{m}+\alpha_{1} \lambda^{m-1}+\cdots
$$

be the minimum polynomial of $d$ over $\Phi$. Then it is clear that $\phi(u)=0$. Hence a necessary condition that $b$ be a $d$-integral is that $\phi(v)=0$. Now

$$
v^{r}=\left(\begin{array}{ll}
d^{r} & B_{r} \\
0 & d^{r}
\end{array}\right)
$$

where $B_{r}=\sum_{k=0}^{r-1} d^{k} b d^{r-k-1}$. Hence the condition that $\phi(v)=0$ is that

$$
B_{m}+\alpha_{1} B_{m-1}+\cdots+\alpha_{m-1} B_{1}=0 .
$$

As we have shown elsewhere ${ }^{3}$

$$
B_{r}=C_{r, 1} d^{r-1} b+C_{r, 2} d^{r-2} b^{\prime}+\cdots+b^{(r-1)}
$$

where $b^{(k)}=\left(b^{(k-1)}\right)^{\prime}$. Hence if we define

$$
\phi_{h}(\lambda)=C_{m, h} \lambda^{m-h}+C_{m-1, h} \alpha_{1} \lambda^{m-h-1}+\cdots \equiv \phi^{(h)}(\lambda) / h !
$$

we may write (4) in the more useful form

$$
\phi_{1}(d) b+\phi_{2}(d) b^{\prime}+\cdots+\phi_{m}(d) b^{(m-1)}=0 .
$$

We suppose now that $\phi_{1}(\lambda) \equiv \phi^{\prime}(\lambda)$ is relatively prime to $\phi(\lambda){ }^{4}$ Then $\phi_{1}(d)$ is a regular element in $\mathfrak{A}$. Hence if $b$ is an element such that (5) holds,

$$
b=-\phi_{1}(d)^{-1} \phi_{2}(d) b^{\prime}-\cdots-\phi_{1}(d)^{-1} \phi_{m}(d) b^{(m-1)}=x^{\prime}
$$

where

$$
x=-\phi_{1}(d)^{-1} \phi_{2}(d) b-\cdots-\phi_{1}(d)^{-1} \phi_{m}(d) b^{(m-2)} .
$$

8 Loc. cit. footnote 1, p. 209.

- This condition will be satisfied if $d$ generates a separable algebraic field over $\Phi$. 
This proves the following theorem.

THEOREM 1. Let $\mathfrak{A}$ be an arbitrary algebra and let $d$ be an algebraic element of $\mathfrak{A}$ having a minimum polynomial $\phi(\lambda)$ relatively prime to its derivative. Then (5) is a necessary and sufficient condition in order that the element $b$ be a d-integral. When the condition holds, $b=x^{\prime}$ where $x$ is given by (6).

We suppose now that $\mathfrak{A}$ is a simple algebra with an identity satisfying the descending chain condition for left (right) ideals. Then $\mathfrak{A}=\mathfrak{D}_{h}$, a matrix algebra of $h$ rows over the (not necessarily finite) division algebra $\mathfrak{D}$ and conversely any algebra of this form satisfies our condition. As before let $d$ be an algebraic element of $\mathfrak{A}$ and let $\phi(\lambda)$ be its minimum polynomial. Let $b$ be an element of $\mathfrak{A}$ such that (5) holds. Then (4) holds and hence the minimum polynomial of $v$ as well as of $u$ is $\phi(\lambda)$. Since $d, b \in \mathfrak{A}=\mathfrak{D}_{h}$ the matrices $u$ and $v \in \mathfrak{D}_{2 h}$ and these may be regarded as the matrices of linear transformations in a $2 h$-dimensional vector space $\Re$ over $\mathfrak{D}$. Let $T$ be the linear transformation corresponding to $v$. Then according to the form of $v$ we have an $h$-dimensional subspace $\subseteq$ of $\Re$ invariant under $T$ such that the matrix of $T$ in $\subseteq$ is $d$ and the matrix of $T$ in the difference space $\Re-\mathfrak{S}$ is also $d$.

We suppose now that $\phi(\lambda)$ is a product of irreducible factors in $\Phi[\lambda]$. In this case the linear transformation is completely reducible. ${ }^{5}$ Hence there exists a subspace $\mathscr{S}^{\prime}$ invariant under $T$ such that $\Re=\mathfrak{S}+\mathfrak{S}^{\prime}$, $\mathfrak{S} \cap \mathfrak{S}^{\prime}=0$ and such that the matrix of $T$ in $\mathfrak{S}^{\prime}$ is also $d$. Let $x_{1}, \cdots, x_{h}, x_{h+1}, \cdots, x_{2 h}$ be the original basis of $\Re$ relative to which $T$ has the matrix $v$ so that $x_{1}, \cdots, x_{h}$ is a basis for $\subseteq$. Corresponding to the decomposition $\Re=\mathfrak{S}+\mathfrak{S}^{\prime}$ we have the basis $x_{1}, \cdots, x_{h}, x_{h+1}^{\prime}, \cdots, x_{2 h}^{\prime}$. The matrix relating this basis to the original one has the form

$$
\left(\begin{array}{ll}
1 & p \\
0 & q
\end{array}\right)
$$

where $p, q \in \mathfrak{D}_{h}$ and the matrix of $T$ relative to the basis $x_{1}, \cdots, x_{h}$, $x_{h_{+1}^{\prime}}^{\prime}, \cdots, x_{2 h}^{\prime}$ is $u$. Hence we have the equation

$$
\left(\begin{array}{ll}
d & 0 \\
0 & d
\end{array}\right)=\left(\begin{array}{ll}
1 & p \\
0 & q
\end{array}\right)^{-1}\left(\begin{array}{ll}
d & b \\
0 & d
\end{array}\right)\left(\begin{array}{ll}
1 & p \\
0 & q
\end{array}\right) .
$$

This implies that $d q=q d$ and that $b q=p d-d p$. Since the matrix

\footnotetext{
${ }^{5}$ See the author's paper Pseudo-linear transformations, Ann. of Math. vol. 38 (1937) p. 498.
} 


$$
\left(\begin{array}{ll}
1 & p \\
0 & q
\end{array}\right)
$$

is regular, $q$ is regular and hence we have the relation $b=x d-d x$ where $x=p q^{-1}$.

THEOREM 2. Let $\mathfrak{A}=\mathfrak{D}_{h}$ where $\mathfrak{D}$ is a division algebra over $\Phi$ and let $d$ be an algebraic element of $\mathfrak{A}$. Then if the minimum polynomial $\phi(\lambda)$ of $d$ is a product of distinct irreducible factors in $\Phi[\lambda]$, the condition (5) is necessary and sufficient in order that the element $b$ of $\mathfrak{A}$ be $a$ d-integral.

We next let $\mathfrak{U}=\Phi_{h}$, the matrix algebra of $h$ rows over $\Phi$. Let $d$ be a non-derogatory matrix in $\Phi_{h}$. Thus $d$ has only one invariant factor $\phi(\lambda) \neq 1$ and $\phi(\lambda)$ is the minimum polynomial of $d$. Let $b$ be a matrix such that (5) holds and consider the matrix $v$ as before. The minimum polynomial of $v$ is $\phi(\lambda)$. If $T$ is the linear transformation in the $2 h$-dimensional space over $\Phi$ associated with the matrix $v$ then $\Re$ contains an invariant subspace $\subseteq$ whose matrix is $d$. Since $d$ is nonderogatory, $\subseteq$ is a cyclic subspace and its order is the minimum polynomial of $T$ in $\Re$. Now it is known that this implies that $\Re=\mathfrak{S}+\mathfrak{S}^{\prime}$, $\Im \cap \varsigma^{\prime}=0$ where $\widetilde{S}^{\prime}$ is also invariant relative to $T .{ }^{6} \mathrm{~A}$ repetition of the argument used to prove Theorem 2 will now yield the following theorem.

THEOREM 3. Let $d$ be a non-derogatory matrix in the matrix algebra $\Phi_{h}$ and let $\phi(\lambda)$ be its minimum polynomial. Then the condition (5) is necessary and sufficient that the matrix $b$ be a d-integral.

We give finally an example in which the condition (5) is not sufficient to insure that an element be a $d$-integral. Let

$$
d=\left(\begin{array}{lll}
0 & 1 & 0 \\
0 & 0 & 0 \\
0 & 0 & 0
\end{array}\right), \quad b=\left(\begin{array}{lll}
0 & 0 & 0 \\
0 & 0 & 0 \\
0 & 0 & 1
\end{array}\right) .
$$

Then the minimum polynomial of $d$ is $\phi(\lambda)=\lambda^{2}$. Since $b d=d b=0$, $b$ satisfies (5). On the other hand, the invariant factors of the matrices $u$ and $v$ here are respectively $\lambda^{2}, \lambda^{2}, \lambda, \lambda$ and $\lambda^{2}, \lambda^{2}, \lambda^{2}$. It follows that these matrices are not similar and hence $b$ is not a $d$-integral.

ThE JohNS Hopkins UNIVERSITY

- See van der Waerden's Moderne Algebra, vol. 2, pp. 129-130. The proof given there of this theorem for ordinary finite groups is also valid for vector spaces relative to a single linear transformation. 